\title{
A Mobicast Routing Protocol with Carry-and-Forward in Vehicular Ad-Hoc Networks
}

\author{
Yuh-Shyan Chen \\ Department of CSIE \\ National Taipei University, Taiwan \\ Email: yschen@mail.ntpu.edu.tw
}

\author{
Yun-Wei Lin and Sing-Ling Lee \\ Department of CSIE \\ National Chung Cheng University, Taiwan \\ Email: jyneda@gmail.com, singling@cs.ccu.edu.tw
}

\begin{abstract}
In vehicular networks, safety and comfort applications are two quite different kinds of applications to avoid the emergency traffic accident and enjoy the non-emergency entertainment. The comfort application drives the challenges of new non-emergency entertainments for vehicular ad-hoc networks (VANETs). The comfort application usually keeps the delaytolerant capability; that is, messages initiated from a specific vehicle at time $t$ can be delivered through VANETs to some vehicles within a given constrained delay time $\lambda$. In this paper, we investigate a new mobicast protocol to support comfort applications for a highway scenario in vehicular ad-hoc networks (VANETs). All vehicles located in a geographic zone (denoted as zone of relevance, ZOR) at time $t$, the mobicast routing is to disseminate the data message initiated from a specific vehicle to all vehicles which have ever appeared in ZOR at time $t$. This data dissemination must be done before time $t+\lambda$ through the carry-and-forward technique. In addition, the temporary network fragmentation problem is considered in our protocol design. In addition, the low degree of channel utilization is kept to reserve the resource for safety applications. To illustrate the performance achievement, simulation results are examined in terms of message overhead, dissemination successful rate, and accumulative packet delivery delay.
\end{abstract}

Index Terms-vehicular ad hoc network, carry-and-forward, mobicast, routing.

\section{INTRODUCTION}

The vehicular ad hoc network (VANET) is the promising techniques for building the ITS. Recently, a new multicast communication paradigm called a "spatiotemporal multicast" or "mobicast" was investigated in [1] which support spatiotemporal coordination in safety applications for VANETs. The distinctive feature of mobicast is to deliver a message at time $t$ to all vehicles located in a prescribed region before time $t+1$. The delivery delay of the message should be short because of the safety purpose. On the other hand, the mobicast routing protocol is also able to develop for region-relevant comfort applications on VANETs. The purpose of region-relevant comfort applications is to deliver a message or multimedia file initiated from a specific vehicle $V$ to all vehicles which have appeared in a prescribed region at time $t$, where the prescribed region is near to vehicle $V$. Comfort applications usually keep the delay-tolerant capability. Messages can deliver to some vehicles within a constrained delay time. Assuming that $\lambda$ is the constrained delay time. That is, the mobicast routing protocol for region-relevant comfort applications is to deliver a message to vehicles which have ever appeared in a prescribed region at time $t$ before time $t+\lambda$. For example, a driver can send a multimedia file to other vehicles appeared in a prescribed region at time $t$, and those vehicles should receive the file before time $t+\lambda$. In addition, the message delivery should maintain a low degree of channel utilization to reserve the resource for safety applications.

In this paper, a new mobicast protocol with carry-andforward is proposed for a highway scenario to support the region-relevant comfort applications. This prescribed region is a geographic zone and is denoted as zone of relevance (ZOR) which vehicles in this region should receive the mobicast message. The spatiotemporal characteristic of a mobicast for comfort applications is to disseminate a mobicast message to all mobile vehicles which have ever appeared in the ZOR at time $t$, and those vehicles must receive the mobicast message before time $t+\lambda$. Observe that, a vehicle easily moves out of the communication range of the other vehicles in a highway scenario and fails to receive mobicast messages. This condition called as temporary network fragmentation problem. Joshi et al. [2] proposed a distributed robust geocast protocol (DRG) to deliver a message to vehicles located in the ZOR and overcome the temporary network fragmentation problem by using the ZOF which is defined in [2]. ZOF is a geographic region which vehicles in this region should forward the geocast message to other vehicles in the ZOR. However, a fixed size of ZOF is difficult to handle the rapid changed topology and easily wastes the unnecessary channel resource. The main contribution of our work is to achieve high dissemination successful rate and maintain a low degree of channel utilization; meanwhile, the delivery delay should be reduced as much as possible.

The rest of this paper is organized as follows. Section II presents the challenges and basic ideas. Section III presents the new mobicast routing protocol. Performance analysis is discussed in Section IV. Finally, Section V concludes this paper.

\section{Preliminaries}

\section{A. System Model}

To successfully deliver the mobicast message to vehicles which have ever appeared in the ZOR at time $t$ before time $t+\lambda$, the temporary network fragmentation problem should be considered. In this work, a message is carried by some possible vehicles to forward to overcome the temporary network fragmentation problem and maintain a low degree of channel utilization at the same time. In a VANET, a vehicle is said as an event vehicle or $V_{e}$ if a comfort application is triggered by a user. $V_{e}$ send the mobicast message to other 
vehicles have appeared in the ZOR at time $t$. In the following, we define $Z \mathrm{R}_{t}$ (zone of relevance) to indicate which vehicle should receive the mobicast message and $\mathrm{ZOF}_{t+i}$ (zone of forwarding) to indicate which vehicle should carry the mobicast message to forward. Let $m_{t}$ denote as a mobicast message sent at time $t$ and $V_{j}$ denote as the vehicle ID, where $j=\{e, 1, \ldots, j, \ldots, n\}$ throughout this paper. Event vehicle $V_{e}$ is the mobicast-initiated vehicle which initiates a mobicast routing protocol to disseminate the mobicast messages $m_{t}$ to other vehicles which have appeared in the $\mathrm{ZOR}_{t}$ before time $t+\lambda$.

Definition 1: $\mathbf{Z O R}_{t}$ (zone of relevance): Given an event vehicle $V_{e}$ and a constrained delay time $\lambda, Z \mathrm{ZR}_{t}$ is a static elliptic region determined by $V_{e}$ at time $t$, such that vehicle $V_{j}$ must be successfully received the mobicast message $m_{t}$ from $V_{e}$ before time $t+\lambda$, where each $V_{j}$ has ever appeared in the $\mathrm{ZOR}_{t}$.

Considering the characteristic of VANETs, $Z_{t}$ is an elliptic region, which has been proofed in [1]. Let $V_{j}^{t}$ denote as a vehicle which has ever appeared in the $\mathrm{ZOR}_{t}$ and should receive the mobicast message $m_{t}$. Fig. 1 (a) shows an example of $\mathrm{ZOR}_{t} . V_{e}$ initiated a $\mathrm{ZOR}_{t}$ and the transmission range of each vehicle is assumed $r . V_{1}^{t}, V_{2}^{t}, V_{3}^{t}$, and $V_{4}^{t}$ have appeared in the $\mathrm{ZOR}_{t}$ and should receive the mobicast message $m_{t}$ before time $t+\lambda$. However, $V_{4}^{t}$ is out of the communication range of other vehicles and encounters the temporary network fragmentation problem. To overcome the temporary network fragmentation problem, $\mathrm{ZOF}_{t+i}$ is used to indicate which vehicle should carry $m_{t}$ and forward to $V_{j}^{t}$. The formal definition of $\mathrm{ZOF}_{t+i}$ is given.

Fig. 2 gives an example of mobicast, which the constrained delay time assumes $\lambda=2$. Initially, $V_{e}$ triggers an event at time $t$ to form a $\mathrm{ZOR}_{t} . V_{1}^{t}, V_{2}^{t}, V_{3}^{t}$, and $V_{4}^{t}$ have appeared in the $\mathrm{ZOR}_{t}$ and should receive $m_{t}$ before time $t+2 . V_{e}$ directly transmits the mobicast message to $V_{1}^{t}$ and $V_{2}^{t} \cdot V_{3}^{t}$ and $V_{4}^{t}$ are out of communication range of other vehicles; hence, they cannot receive $m_{t} . V_{3}^{t}$ and $V_{4}^{t}$ encounter the temporary network fragmentation problem. At time $t+1, V_{2}^{t}$ carries $m_{t}$ and moves closed to $V_{3}^{t}$, then $V_{2}^{t}$ forwards $m_{t}$ to $V_{3}^{t}$. At time $t+2, V_{1}^{t}$ carries $m_{t}$ and moves closed to $V_{4}^{t}$, then $V_{1}^{t}$ forwards $m_{t}$ to $V_{4}^{t}$. Observe that $V_{5}$ has not appeared in $\mathrm{ZOR}_{t}$ at time $t$, therefore $V_{5}$ does not receive $m_{t}$.

Definition 2: $\mathbf{Z O F}_{t+i}$ (zone of forwarding): Given a $V_{e}$, $\mathrm{ZOF}_{t+i}$ is a geographic region determined at each time $t+i$, where $i=0,1, \ldots, i$, such that each vehicle $V_{j}$ has the responsibility of carrying and forwarding the mobicast message $m_{t}$, where $V_{j}$ is located in the $\mathrm{ZOF}_{t+i}$.

In this works, $\mathrm{ZOF}_{t+i}$ is separated by $V_{e}$ 's position into two sub-zones, the rear sub-zone and the front sub-zone. Let $\mathrm{ZOF}_{t+i}^{R}$ denote as the rear sub-zone behind $V_{e}$. Let $\mathrm{ZOF}_{t+i}^{F}$ denote as the front sub-zone, which is in front of $V_{e}$. This is, $\mathrm{ZOF}_{t+i}=\mathrm{ZOF}_{t+i}^{R} \cup \mathrm{ZOF}_{t+i}^{F}$. The center location of $\mathrm{ZOF}_{t+i}$ is the same with the location of $V_{e}$, moving at the same speed as $V_{e}$, and toward the same direction with $V_{e}$. Fig. 1 (b) shows $V_{1}^{t}, V_{2}^{t}, V_{3}^{t}$, and $V_{4}^{t}$ located in $\mathrm{ZOF}_{t+i}$; hence $V_{2}^{t}$ should directly forward $m_{t}$ to $V_{3}^{t}$ and $V_{1}^{t}$ should carry $m_{t}$ to forward to $V_{4}^{t}$.

\section{B. Basic Idea}

The method to deliver $m_{t}$ can be divided into two techniques, multihop forwarding and carry-and-forward tech-
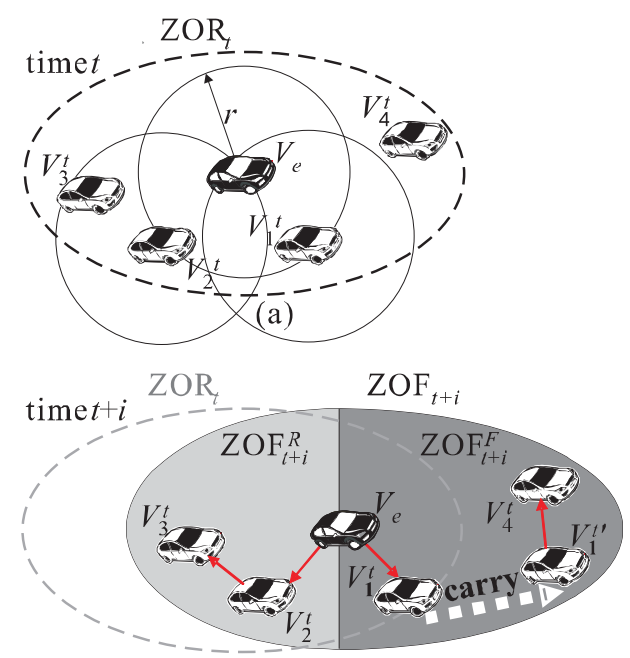

(b)

Fig. 1. (a) $\mathrm{ZOR}_{t}$ (zone of relevance) and (b) $\mathrm{ZOF}_{t+i}$ (zone of forwarding) is the union set of $\mathrm{ZOR}_{t} \cup \mathrm{ZOF}_{t+i}^{R} \cup \mathrm{ZOF}_{t+i}^{F}$.
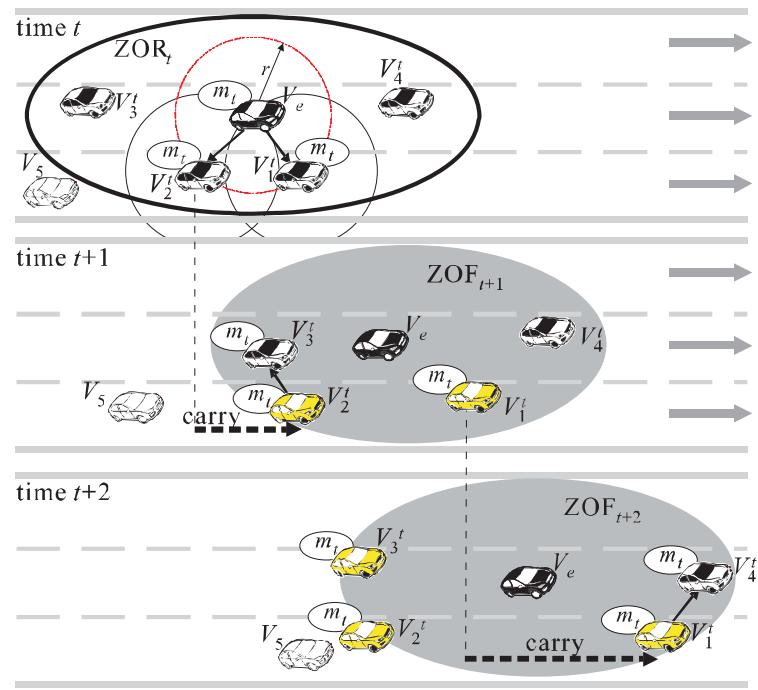

Fig. 2. An example of mobicast routing with carry-and forward.

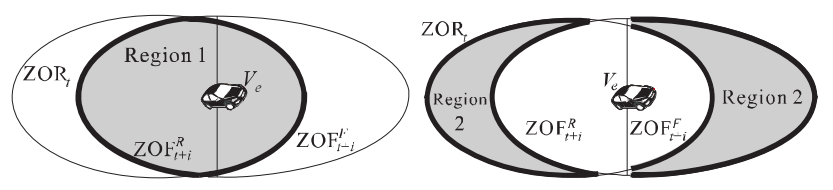

(a)

(b)

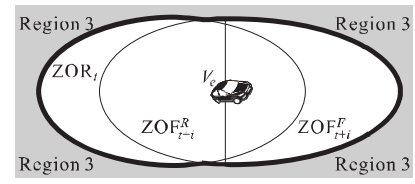

(c)

Fig. 3. (a) region 1 , (b) region 2, and (c) region 3 


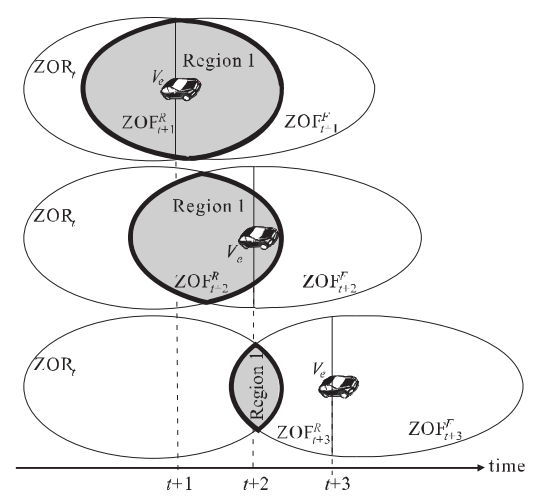

Fig. 4. The area of region 1 is decreasing as the time increasing.

niques. The multihop forwarding technique delivers $m_{t}$ to $V_{j}^{t}$ by multihop wireless transmission. The channel utilization is high and the delivery delay is short. The carry-and-forward technique delivers $m_{t}$ to $V_{j}^{t}$ by some possible vehicles carrying and forwarding. The channel utilization is low but the delivery delay is long. Comfort applications usually keep the delay-tolerant capability and the channel resource should be reserved for safety application. Hence, the mobicast routing for comfort applications should use the carry-and-forward technique as far as possible. The multihop forwarding technique is only used when it is necessary. The key problem in this work is how to choose the message delivery technique between multihop forwarding and carry-and-forward techniques to achieve high dissemination successful rate and maintain a low degree of channel utilization; meanwhile, the delivery delay should be reduced as much as possible.

In this work, the message delivery techniques are chosen according to the region. Three regions can be identified as shown in Fig 3 because $Z^{2 O R}$ is a static region and $\mathrm{ZOF}_{t+i}$ is constantly moving with $V_{e}$. Region 1 is $\mathrm{ZOR}_{t} \cap($ $\left.\mathrm{ZOF}_{t+i}^{R} \cup \mathrm{ZOF}_{t+i}^{F}\right)$. Region 2 is $\left(\mathrm{ZOR}_{t} \cup \mathrm{ZOF}_{t+i}^{R} \cup \mathrm{ZOF}_{t+i}^{F}\right)-$ region 1. Region 3 is ${ }^{\sim}\left(\mathrm{ZOR}_{t} \cup \mathrm{ZOF}_{t+i}^{R} \cup \mathrm{ZOF}_{t+i}^{F}\right)$. The key idea of this work is to deliver the mobicast massage by using multihop forwarding technique in region 1 , and using carryand-forward technique in region 2. Considering the trajectory of vehicles, all vehicles are continuously moving ahead. Both region 1 and region 2 can cover $V_{j}^{t}$. However, region 1 can cover $V_{j}^{t}$ more easily than region 2 . The multihop forwarding technique is used in region 1 because it is necessary to use the wireless transmission to deliver the mobicast message $m_{t}$ to $V_{j}^{t}$. But compared to region 1 , region 2 also covers some other vehicles which do not need to receive the mobicast message. That is the reason why the carry-and-forward technique is used in region 2. For those vehicles which do not need to receive the mobicast message in region 2, there is unnecessary for them to get involved in delivering messages. Therefore, the channel resource can be reserved. There is an exception which the multihop forwarding technique is used in region 2 if the mobicast message can not deliver to destined vehicles before time $t+\lambda$. In addition, the mobicast message $m_{t}$ is dropped in region 3 because region 3 does not cover $V_{j}^{t}$.

Observe that, the area of region 1 is decreasing as time goes by because $\mathrm{ZOF}_{t+i}$ is constantly moving with $V_{e}$ as shown in Fig 4. Initially, the area of region 1 is large. The multihop

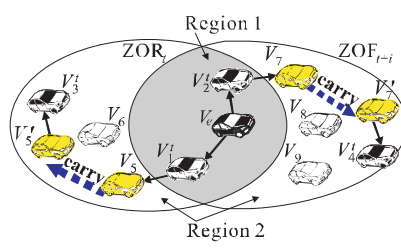

(a)

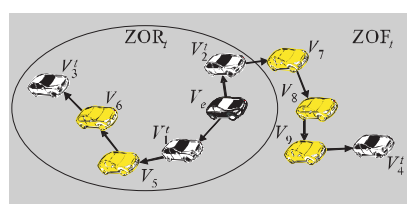

(b)
Fig. 5. Protocol comparison: (a) mobicast routing protocol with carry-andforward and (b) distributed robust geocast protocol.

forwarding technique is used in region 1 to deliver $m_{t}$ for most of vehicles $V_{j}^{t}$ with short delivery delay. The delivery delay can be reduced as much as possible. As time goes by, vehicles using multihop forwarding technique to deliver the mobicast message are becoming fewer and fewer in region 1. Vehicles use carry-and-forward technique to deliver the mobicast message $m_{t}$ and overcome the temporary network fragmentation problem in region 2 . Hence, more and more channel resource are reserved for safety applications. That is, our mobicast routing protocol reduces the delivery delay time as much as possible, maintains a low degree of channel utilization, and achieves the high dissemination successful rate by overcoming the temporary network fragmentation problem. Fig. 5 (a) shows that our mobicast routing protocol only uses multihop forwarding technique in region 1, and carry-andforward technique is used to reserve channel resource in region 2. $V_{6}, V_{8}$, and $V_{9}$ are not involved to deliver mobicast message in our protocol. Compared to DRG [2] as shown in Fig. 5 (b), our protocol can reserve more channel resource.

\section{Mobicast Routing Protocol}

The mobicast routing protocol is split into three phases: (1) $\mathrm{ZOR}_{t}$ creation phase, (2) $\mathrm{ZOF}_{t+i}$ estimation phase, and (3) message dissemination phase. The detailed operation is developed as follows.

\section{A. ZOR $_{t}$ Creation Phase}

In this paper, we assume that each vehicle can acquire location information via location information providers, such as the Global Positioning System. Let $L_{t}^{V_{j}}=\left(x_{t}^{V_{j}}, y_{t}^{V_{j}}\right)$ denote as the location of $V_{j}$ at time $t$. Let $v_{j_{t}}$ denote as the velocity $V_{j}$ at time $t$. Each vehicle $V_{j}$ exchanges $L_{t}^{V_{j}}$ and $v_{j_{t}}$ to its neighbors by hello message. The procedure of the $\mathrm{ZOR}_{t}$ creation phase is given herein.

$\mathrm{S} 1 . V_{e}$ announces the $\mathrm{ZOR}_{t}$ which is determined by $Z_{t}\left(L_{t}^{V_{j}}\right)=\frac{\left(x_{t}^{V j}-x_{t}^{V_{e}}\right)^{2}}{a^{2}}+\frac{\left(y_{t}^{V j}-y_{t}^{V_{e}}\right)^{2}}{b^{2}}-1=0$, where $a$ is the major axis of the $\mathrm{ZOR}_{t}$ and $b$ is the minor axis of the $\mathrm{ZOR}_{t}$. Major axis $a$ is determined by the requirement of comfort application and minor axis $b$ is determined by the width of lane.

$\mathrm{S} 2$. The usual velocity of $V_{e}$ is necessary to predict the location of $V_{e}$, which is used to describe the border of $\mathrm{ZOF}_{t+i}$. Let $v_{e}^{h}$ denote as the usual velocity of $V_{e}$. Based on the investigation of [3], the velocity is generally assumed as normal distributed in highway scenario. Each driver usually drives a car with a specific velocity pattern; therefore, $v_{e}^{h}$ can be used to represent the usual velocity of $V_{e}$. Let $v_{j}^{\text {low }}$ and $v_{j}^{\text {upper }}$ denote as the low bound and upper bound velocity of $V_{j}$, respectively. The low 
bound and upper bound velocity of $V_{e}$ are $v_{e}^{\text {low }}=$ $\overline{v_{e}}-t_{\text {Gossett }} \times \frac{\widehat{S}_{v_{e}}}{\sqrt{n}}$ and $v_{e}^{\text {upper }}=\overline{v_{e}}+t_{\text {Gossett }} \times \frac{\widehat{S}_{v_{e}}}{\sqrt{n}}$, where $\overline{v_{e}}$ is the mean velocity of $V_{e}, n$ is the number of recorded velocities, $t_{\text {Gosset }}$ is a parameter under $C I \%$ in Gosset's t-distribution [4], where $C I \%$ denotes the confidence interval of the estimated velocity, and $\widehat{S}_{v_{e}}$ is the standard deviation of $S\left(V_{e}\right)$, where $\widehat{S}_{v_{e}}=\sqrt{\frac{\sum\left(v_{e_{t}}-\overline{v_{e}}\right)^{2}}{n-1}}$. Then, $v_{e}^{h}$ can be acquired form $v_{e}^{\text {low }}$ and $v_{e}^{\text {upper }}$ by harmonic mean computing as $v_{e}^{h}=\frac{1}{\frac{1}{v_{e}^{l o w}}+\frac{1}{v_{e}^{u p p e r}}}$.

S3. The $V_{e}$ broadcasts the mobicast control packet $P_{m}\left(V_{j}\right.$, $\left.v_{e}^{h}, Z_{t}\left(L_{t}^{V_{e}}\right), m_{t}, t_{s}, a\right)$, where $t_{s}$ is sending time when $V_{e}$ sends $P_{m}$. After $V_{e}$ broadcasted the $P_{m}, \mathrm{ZOF}_{t+i}$ creation phase is executed.

\section{B. $Z_{\text {OF }}$ Estimation Phase}

$\mathrm{ZOF}_{t+i}$ is to indicate which vehicle should deliver the mobicast message $m_{t}$ for $V_{j}^{t}$ at each time $t+i$ ZOF $_{t+i}$ consists of $\mathrm{ZOF}_{t+i}^{R}$ and $\mathrm{ZOF}_{t+i}^{F}$. $\mathrm{ZOF}_{t+i}^{R}$ is used to deliver $m_{t}$ to $V_{j}^{t}$ behind $V_{e}$ and $\mathrm{ZOF}_{t+i}^{F}$ is used to deliver $m_{t}$ to $V_{j}^{t}$ in front of $V_{e}$. The detailed operation is developed as follows.

A1. To know the necessary of receiving $m_{t}, V_{j}$ checks whether it has appeared in $\mathrm{ZOR}_{t}$ at time $t$ if $V_{j}$ receives a $P_{m}$. If $Z_{t}\left(L_{t}^{V_{j}}\right) \leq 0, V_{j}$ is $V_{j}^{t}$ and it is necessary for receiveing $m_{t}$, then goto to A2. Otherwise, If $Z_{t}\left(L_{t}^{V_{j}}\right) \geq 0$, goto message dissemination phase.

A2. $V_{j}^{t}$ compares its location with $V_{e}$ to know it is located in eithor $\mathrm{ZOF}_{t+i}^{R}$ or $\mathrm{ZOF}_{t+i}^{F}$ because $\mathrm{ZOF}_{t+i}$ is split by $V_{e}$ 's location. If $\left(x_{t+i}^{V_{j}}-x_{t+i}^{V_{e}}\right)<0, V_{j}^{t}$ is located in $\mathrm{ZOF}_{t+i}^{R}$, then goto $\mathrm{A} 3$, where $x_{t+i}^{V_{e}}=x_{t}^{V_{e}}+v_{e}^{h} \times i$. Otherwise, if $\left(x_{t+i}^{V_{j}}-x_{t+i}^{V_{e}}\right)>0, V_{j}^{t}$ is located in $\mathrm{ZOF}_{t+i}^{F}$, then goto A4.

A3. To deliver $m_{t}$ to all $V_{j}^{t}$ behind $V_{e}, \mathrm{ZOF}_{t+i}^{R}$ is formed to cover all $V_{j}^{t}$ behind $V_{e}$ at each time $t+i$ by estimating the major axis $a_{R}$ of $\mathrm{ZOF}_{t+i}^{R}$. Let $v_{R}^{\text {mean }}$ denote as the mean velocity of all $V_{j}^{t}$ behind $V_{e}$. The major axis $a_{R}$ is computed by $a_{R}=\left(v_{e}^{h}-v_{\text {mean }}^{R}\right) \times i+a$, where $v_{R}^{\text {mean }}=$ $\frac{1}{\frac{1}{v_{j}^{\text {low }}}+\frac{2}{\text { previous }-v_{R}^{\text {mean }}}}$. Observe that, $v_{R}^{\text {mean }}$ is computed by low bound of $V_{j}^{t}$ in order to cover all $V_{j}^{t}$ behind $V_{e}$ as could as possible. Then goto A5.

A4. To deliver $m_{t}$ to all $V_{j}^{t}$ in front of $V_{e}, \mathrm{ZOF}_{t+i}^{F}$ is formed to cover all $V_{j}^{t}$ in front of $V_{e}$ by estimating the major axis $a_{F}$ of $\mathrm{ZOF}_{t+i}^{F}$. Let $v_{F}^{\text {mean }}$ denote as the mean velocity of all $V_{j}^{t}$ in front of $V_{e}$. The major axis $a_{F}$ of $\mathrm{ZOF}_{t+i}^{F}$ is computed by $a_{F}=\left(v_{\text {mean }}^{F}-v_{e}^{h}\right) \times\left(t+i-t_{s}\right)+a V_{j}^{t}$,

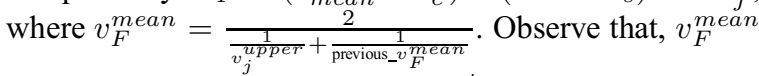
is computed by upper bound of $V_{j}^{t}$ in order to cover all $V_{j}^{t}$ in front of $V_{e}$ as could as possible.

A5. $\mathrm{ZOF}_{t+i}^{R}$ and $\mathrm{ZOF}_{t+i}^{F}$ are formed by $F_{t+i}^{R}\left(L_{t+i}^{V_{j}}\right)=$ $\frac{\left(x_{t+i}^{V j}-x_{t+i}^{V_{e}}\right)^{2}}{a_{R}^{2}}+\frac{\left(y_{t+i}^{V j}-y_{t}^{V_{e}}\right)^{2}}{b^{2}}-1=0$ and $F_{t+i}^{F}\left(L_{t+i}^{V_{j}}\right)=$ $\frac{\left(x_{t+i}^{V j}-x_{t+i}^{V e}\right)^{2}}{a_{F}^{2}}+\frac{\left(y_{t+i}^{V j}-y_{t}^{V e}\right)^{2}}{b^{2}}-1=0$, respectively. Then goto message dissemination phase.

Fig. 6 shows examples of $\mathrm{ZOF}_{t+i}^{R}$ and $\mathrm{ZOF}_{t+i}^{F}$. Observe that, $a_{R}$ and $a_{F}$ are estimated based on $v_{j}^{\text {low }}$ and $v_{j}^{\text {upper }}$, re-

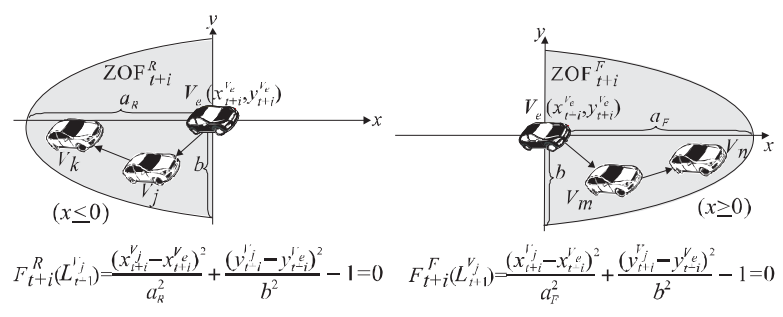

(a)

(b)

Fig. 6. Creation of (a) $\mathrm{ZOF}_{t+i}^{R}$ and (b) $\mathrm{ZOF}_{t+i}^{F}$.

spectively. The purpose is to enhance dissemination successful rate by covering all $V_{j}^{t}$ as far as possible.

\section{Message Dissemination Phase}

In the message dissemination phase, $m_{t}$ is disseminated to all $V_{j}^{t}$. A node chooses eithor multihop forwarding technique or carry-and-forward technique to deliver $m_{t}$ according to the region which the node is located. Besides, $m_{t}$ should be delivered under constrained delay time $\lambda$. The procedure of the message dissemination phase is described below.

$\mathrm{S} 1$. When $V_{j}$ receives a $P_{m}$ at time $t+i, V_{j}$ directly forward $P_{m}$ to neighboring vehicles using multihop forwarding technique if one of the two conditions is true: (C1) $Z_{t}\left(L_{t+i}^{V_{j}}\right) \leq 0$ and $F_{t+i}^{R}\left(L_{t}^{V_{j}}\right) \leq 0$, or $(\mathrm{C} 2) Z_{t}\left(L_{t+i}^{V_{j}}\right) \leq 0$ and $F_{t+i}^{F}\left(L_{t}^{V_{j}}\right) \leq 0$. Both the two conditions represent $V_{j}$ located within region 1 . Condition $\mathrm{C} 1$ implys $V_{j}$ located in both $\mathrm{ZOR}_{t}$ and $\mathrm{ZOF}_{t+i}^{R}$. Condition C2 implys $V_{j}$ located in both $\mathrm{ZOR}_{t}$ and $\mathrm{ZOF}_{t+i}^{F}$. Otherwise goto S2.

S2. $V_{j}$ forward $P_{m}$ to other $V_{j}^{t}$ with carry-and-forward technique if one of the two conditions is true: (C3) $Z_{t}\left(L_{t+i}^{V_{j}}\right) \leq 0$ and $F_{t+i}^{R}\left(L_{t}^{V_{j}}\right)>0$, or $($ C4 $) F_{t+i}^{F}\left(L_{t}^{V_{j}}\right) \leq$ 0 and $Z_{t}\left(L_{t+i}^{V_{j}}\right)>0$. Both the two conditions represent $V_{j}$ located within region 2 . Condition C3 implys $V_{j}$ located in $\mathrm{ZOR}_{t}$ but outside $\mathrm{ZOF}_{t+i}^{R}$. Condition C4 implys $V_{j}$ located in $\mathrm{ZOF}_{t+i}^{F}$ but outside $\mathrm{ZOR}_{t}$. In addition, considering the constrained delay time $\lambda, V_{j}$ still needs to examine the remaind delivery time. Let $R_{j}$ and $F_{j}$ denote as the distance from $V_{j}$ to left border of $\mathrm{ZOR}_{t}$ and right border of $\mathrm{ZOF}_{t+i}^{F}$, respectively. In condition $\mathrm{C} 3$, if $\frac{R_{j}}{v_{j}^{u p p e r}}>\lambda-i, V_{j}$ directly forwards $P_{m}$ to a neighbor vehicle to satisfy the constrained delay time. In condition $\mathrm{C} 4$, if $\frac{F_{j}}{v_{j}^{\text {low }}}>\lambda-i, V_{j}$ directly forwards $P_{m}$ to a neighbor vehicle to satisfy the constrained delay time.

S3. $V_{j}$ drops $P_{m}$ if one condition is true: (C5) $Z_{t}\left(L_{t+i}^{V_{j}}\right)>0$ and $F_{t+i}^{R}\left(L_{t}^{V_{j}}\right)>0$ and $F_{t+i}^{F}\left(L_{t}^{V_{j}}\right)>0$. Condition C5 represent $V_{j}$ located in region 3 .

Fig. 7 shows the mobicast message is delivered by multihop forwarding technique in region 1. Fig. 8 shows the mobicast message is delivered by carry-and-forward technique in region 2 .

\section{Simulation Results}

To evaluate the presented mobicast protocol, our mobicast routing protocol with carry-and-forward (MCF) is simulated and compared to DRG [2]. All these protocols are mainly implemented using the NCTUns 5.0 simulator and emulator 


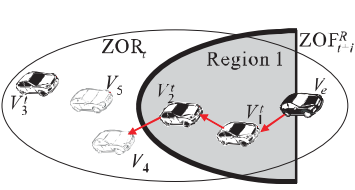

(a)

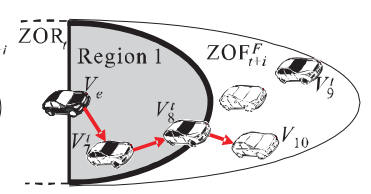

(b)
Fig. 7. The multihop forwarding technique is used in region 1 .

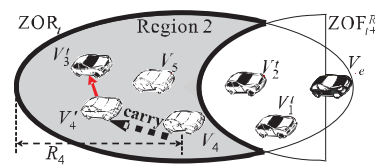

(a)

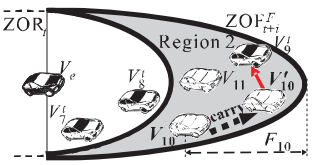

(b)
Fig. 8. The carry-and-forward technique is used in region 2.

[5]. 802.11p protocol is used for physical and MAC layer in this simulation. The system parameters are given below. To discuss the effect of the network density (ND) of a VANET, our simulator considers a $2000 \times 20 \mathrm{~m}^{2}$ highway scenario with various numbers of vehicles, ranging from 40 to 400 . The communication radius of each vehicle is $100 \mathrm{~m}$. The velocity, $v$, of each vehicle is assumed from 10 to $100 \mathrm{~km} / \mathrm{hr}$. The constrained delay time $\lambda$ assumes 35 seconds. Each time unit $i$ assumes $500 \mathrm{~ms}$. $V_{e}$ broadcasts a new $P_{m}$ per minute. The performance metrics to be observed are:

- Message overhead (MO) is the total number of bytes that all vehicles transmitted to deliver the mobicast message.

- Dissemination successful rate (DSR) is the number of vehicles have appeared in $Z O R_{t}$ which can successfully receive the mobicast messages before time $t+\lambda(\lambda=35$ seconds), divided by the total number of vehicles have appeared in $\mathrm{ZOR}_{t}$.

- Accumulative packet delivery delay (APDD) is the accumulative delay time when a mobicast message is received by a vehicle $V_{j}^{t}$.

It is worth mentioning that an efficient mobicast routing protocol in a VANET is achieved with a high DSR, low MO, and low APDD.

\section{A. Message overhead (MO)}

Fig. 9 (a) shows the observed MO under various velocity $v$. A mobicast routing protocol for comfort applications with the lower message overhead implies the degree of channel utilization was low. The channel resource should be reserved for safety applications. Compared to DRG, our mobicast routing protocol with carry-and-forward significantly improves MO.

\section{B. Dissemination successful rate (DSR)}

Fig. 9 (b) shows the observed DSR under various velocity $v$. A mobicast routing protocol with the high dissemination successful rate implies that the value of its DSR was high. In general, the DSR drops as the $v$ increases because the rapid changed topology and frequent happened temporary network fragmentation problem. The temporary network fragmentation problem is frequently occurred when the ND is low. Therefore, DSR was low when ND was low.

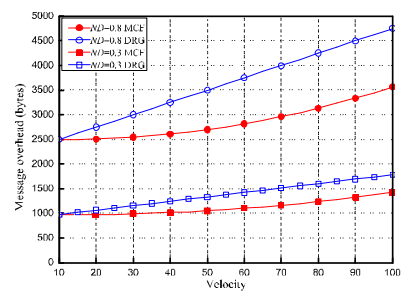

(a)

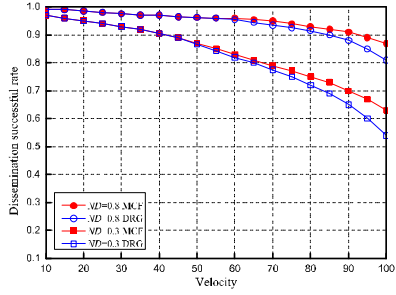

(b)
Fig. 9. Performance of (a) message overhead vs. velocity, (b) dissemination successful rate vs. velocity.

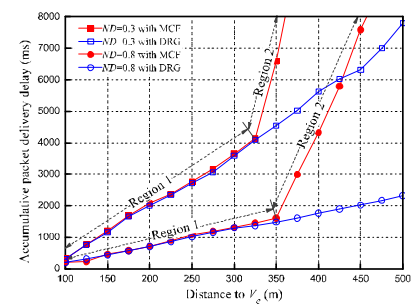

Fig. 10. Performance of accumulative packet delivery delay vs. distance to $V_{e}$.

\section{Accumulative Packet delivery delay (APDD)}

Fig. 10 shows the observed APDD vs. the distance to $V_{e}$. APDD accumulates the deliver delay time for each $V_{j}^{t}$. In general, the APDD increases as the distance increases. Observe that, when the distance to $V_{e} \doteqdot 325 \mathrm{~m}$ under ND $=0.3$ and the distance to $V_{e} \doteq 350 \mathrm{~m}$ under ND $=0.8$, the APDD is intense increasing in our mobicast routing protocol. The is because the message is delivered to region 2 and the carry-and-forward technique is tused.

\section{CONCLUSION}

In this paper, we present a mobicast routing protocol with carry-and-forward to achieve high dissemination successful rate, maintain a low degree of channel utilization and reduce the delivery delay as much as possible.

\section{ACKNOWLEDGEMENTS}

The authors would like to thank the anonymous reviewers and the editors for the valuable suggestions to improve this paper. This research was supported by the National Science Council of the Republic of China (ROC) under grant NSC97-2221-E-305-003-MY3.

\section{REFERENCES}

[1] Y. S. Chen, Y. W. Lin, and S. L. Lee, "A Mobicast Routing Protocol for Vehicular Ad Hoc Networks," ACM/Springer Mobile Networks and Applications (MONET), vol. 15, no. 1, pp. 20-35, 2010.

[2] H. P. Joshi, M. L. Sichitiu, and M. Kihl, "Distributed Robust Geocast: A Multicast Routing for Inter-Vehicle Communication," the First Workshop on WiMAX, Wireless and Mobility, May 2007.

[3] W. Schnabel and D. Lohse, "Grundlagen der Strasenverkehrstechnik und der Verkehrsplanung," pp. Bd. 1, 2. Aufl., Verlag fur Bauwesen, Berlin, 1997.

[4] W. S. Gosset, "The Probable Error of a Mean," Biometrika, vol. 6, no. 1, pp. 1-25, 1908.

[5] S. Wang and C. Lin, "NCTUns 5.0: A Network Simulator for IEEE 802.11(p) and 1609 Wireless Vehicular Network Researches," IEEE International Symposium on Wireless Vehicular Communications, 2008. 